\title{
Hodgkin Lymphoma During Pregnancy
}

National Cancer Institute

\section{Source}

National Cancer Institute. Hodgkin Lymphoma During Pregnancy. NCI Thesaurus. Code C114783.

Hodgkin lymphoma that occurs during pregnancy. 